\title{
Electrospinning polypropylene with an amino acid as a strategy to bind the antimicrobial peptide Cys-LC-LL-37
}

\author{
Frederico Nogueira ${ }^{1,3}$ (D) Pilar Teixeira ${ }^{2}$, and Isabel C. Gouveia ${ }^{3, *}$ \\ ${ }^{1}$ CICS-UBI - Health Sciences Research Centre, University of Beira Interior, Covilhã, Portugal \\ ${ }^{2}$ Institute for Biotechnology and Bioengineering (IBB), Braga, Portugal \\ ${ }^{3}$ FibEnTech R\&D Unit Textile and Paper Materials, Fiber Materials and Environmental Technologies, University of Beira Interior, Rua \\ Marquês d'Ávila e Bolama, 6201-001 Covilhã, Portugal
}

Received: 13 July 2017

Accepted: 20 November 2017

Published online:

27 November 2017

(C) Springer Science+Business Media, LLC, part of Springer Nature 2017

\begin{abstract}
Hospital isolation gowns are increasingly competitive, with brands and manufacturers contesting consumer preferences. The textile materials in contact with the skin can acquire secretions and multiresistant microorganisms, causing discomfort and health risks, respectively. A new nanofibrous substratepolypropylene grafted with L-Cys-was developed with an increased crystallinity, providing its surface with - $\mathrm{SH}$ hooks necessary to efficiently cross-link the antimicrobial peptide Cys-LC-LL-37 in order to protect against nosocomial pathogens and their spread to community. Furthermore, this application does not require environmental control of humidity, and it is not susceptible to enzyme and microorganism degradation.
\end{abstract}

\section{Introduction}

Textiles are considered an attractive substrate for bacteria because of their rich nutrient content, which can function as a reservoir for bacterial infections, especially under optimal conditions of humidity and temperature. In a hospital setting, patients and clinical staff can acquire these bacteria through direct contact with contaminated textiles or their surroundings [1]. When infected in this way, they can transmit the infectious agent with ease to co-workers and patients, which is potentially dangerous if the latter are immunocompromised [2].
Synthetic fibers account for about $62.1 \%$ of the world textile market [3]. In 2015, the production of synthetic fibers roundabout 60.7 million tonnes compared to 6.1 million tonnes of cellulosic fibers. Between 2000 and 2015, the synthetic fibers production has increased $[4,5]$ due in large part to the enhancement of properties such as wetting and hydrophilicity [6, 7]. From 1960s, polypropylene began to conquer the markets and to become a serious competitor of natural and cellulosic fibers, due to its wrinkle-resistant properties, good mechanical strength and fast drying [8]. Polypropylene is composed by saturated linear fibers of aliphatic

Address correspondence to E-mail: igouveia@ubi.pt 
hydrocarbons, and each carbon atom possesses one methyl ramification. It has a low density of $0.90 \mathrm{~g} /$ $\mathrm{cm}^{3}$ and a crystallinity between 45 and $60 \%$, the chains of which present a helical form and group through van der Waals interactions. It has interesting properties, such as good thermobinding properties, smoothness, humidity transfer by capillarity and low cost of production. On the other side, its elasticity is inferior to polyamides. Usually, polypropylene is melted and extruded into filaments, which are then stretched, oriented and stabilized [9]. In fact, most polypropylene textiles are not fibers directly produced by extrusion, but yarns of tapes (strips or filmstrips) prepared from wider films or extruded tapes through rows with elongated holes. There are some works related with the modification of polypropylene. These modifications can be done physically during the manufacturing process, or chemically combining with other copolymers or chemicals. These methods can unfold the internal structure of the fiber, which is distinguished by its low tendency to pilling formation, and may leave modified polypropylene with a different behavior than native polypropylene during dyeing or finishing procedures [5].

The use of antimicrobial textiles is an important step in the fight against a range of hospital pathogens. They should kill a broad spectrum of harmful and antibiotic-resistant bacteria, prevent the bacterial adhesion or biofilm development and not cause skin irritation. Nosocomial infections are associated to pathogenic microorganisms that are easily spread, with Gram-positive Staphylococcus aureus and Gramnegative Pseudomonas aeruginosa generally regarded as the most hazardous [10].

Both S. aureus and P. aeruginosa infections are primarily opportunistic, i.e., they seldom cause diseases in a healthy immune system, but seek to exploit any weaknesses in the body of immunocompromised patients, such as victims of burns, cancer patients or cystic fibrosis, in order to cause infections. Both strains have the ability to grow in any environment, present important virulence factors and have resistance to a large number of antibiotics [11, 12].

In recent years, because of the increasing resistance of bacteria against the antibiotics, quaternary ammonium compounds, silver, polyhexamethylene biguanides and triclosan have been tested, although with limited success. They have powerful bactericidal activity as indicated for its MIC value (minimum inhibition concentration). However, the majority have a reduced spectrum of microbial inhibition and may cause skin irritation, ecotoxicity and bacterial resistance. In addition, its incorporation in textiles substantially slows their performance and limits their accessibility to microorganisms. Moreover, these compounds lose their activity particularly during the intended use and after some washes. For this reason, large amounts of these biocides are applied to textiles to efficiently control the bacterial growth and to keep their durability [13].

To overcome these disadvantages, natural compounds mostly chitosan, hyaluronan and plant extracts are being tested as antimicrobial agents for textiles [14-17]. Also, to fulfill the customers demand for hygienic clothes, and furthermore, the growing market of medical and bioactive textiles has stimulated the research and development of new antimicrobial textiles.

Based on careful literature review, our innovative strategy to develop new antimicrobial textiles considers the utilization of natural defensive amino acids and antimicrobial peptides (AMPs) that are found in every living organism. The organisms from the elementary to most complexes, including humans, produce AMPs against microorganisms [18]. Natural antimicrobial substances have a high structural diversity and a broad spectrum of activity that includes Gram-positive and Gram-negative bacteria, fungi, and in particular cases, some viruses, with the additional advantage of not displaying cytotoxicity or ecotoxicity. Many of these substances are of peptide nature, rich in L-cysteine (L-Cys) residues [18].

The use of L-Cys and AMPs in cosmetic industry and their natural presence in the skin, hair and nails of human beings offers a guarantee of biocompatibility and non-toxicity once in prolonged contact with the body. These guarantees were already proven by our recent results regarding the biofunctionalization of cotton [19, 20], silk textiles [21, 22] and polycaprolactone foam grafted with L-Cys [23], along with a low MIC value, high durability and safety both for user and environment. In general, cationic AMPs with the best antimicrobial activity are those that have the charged and hydrophilic portions to either side with a hydrophobic portion inbetween [24]. In accordance with this, cathelicidin peptides range in length from 12 to 80 residues and may have $\alpha$-helical, $\beta$-sheet or other types of tertiary structures [25]. Likewise, Cys-LC-LL-37 antimicrobial peptide has a 
broad spectrum, namely against Gram-positive $S$. aureus and Gram-negative P. aeruginosa.

This work aims to investigate the potential of natural compounds-L-Cys and Cys-LC-LL-37-as effective, durable and non-toxic antimicrobial agents with the potential for development of biomedical textiles. A new process for biofunctionalization of polypropylene textile-based material and its assessment of the biocidal activity, stability, durability and toxicity was investigated.

\section{Experimental}

Polypropylene (PP) was grafted with L-cysteine (L-Cys) (Sigma). The resulting blend was threaded on electrospinning and subsequently cross-linked with Cys-LC-LL-37 (AnaSpec). S. aureus was cultured on nutrient agar (Panreac) and nutrient broth (Panreac) at $37^{\circ} \mathrm{C} 110 \mathrm{rpm}$; P. aeruginosa PA25 (DSM 25642) was cultured on brain heart infusion (BHI) agar and brain heart infusion (BHI) broth (Sigma) at $30{ }^{\circ} \mathrm{C}$ and $200 \mathrm{rpm}$.

\section{Development of PP-g-SH substrates}

\section{Surface-active bulk additive grafting of PP with L-Cys}

PP grafted with L-Cys (PP-g-SH) (80:20) was dissolved in trifluoroacetic acid (TFA) and dichloromethane (DCM), and stirred at $80^{\circ} \mathrm{C}$ overnight. Since PP is extremely nonpolar, and dissolution follows the rule "like dissolves like," PP was dissolved in DCM, a nonpolar protic solvent with a low dielectric constant of $9.08(<15)$ [26]. TFA, with $\mathrm{F}^{-}$as nucleophile, is also miscible with hydrocarbons and a versatile solvent for materials stable in acid [27], such as PP.

\section{Electrospinning}

Nanofibers of PP (used as a control) and of PP-g-SH (80:20) were threaded from the NanoSpider apparatus (Elmarco s.r.o. Liberec, Czech Republic). The chosen proportion $15 \%(\mathrm{w} / \mathrm{v}) \mathrm{PP} /$ solvent was selected according to the homogeneity and easy detachment from the collector coating. The operating parameters were: voltage $=80 \mathrm{kV}$, droplet-collector distance $=16 \mathrm{~cm}$ and electrode $\mathrm{spin}=7 \mathrm{r} / \mathrm{min}$. The environmental parameters were: temperature $=25^{\circ} \mathrm{C}$ and humidity at $20 \%$.

\section{Washing cycles}

In order to evaluate the stability of the thiol group and if it resisted to laundry, PP-g-SH samples were subjected to 25 washing cycles each in fresh $\mathrm{ddH}_{2} \mathrm{O}$, according to procedure adapted from international standard EN ISO 105-C06:2010.

\section{Characterization}

Fourier transform infrared spectroscopy (FT-IR) The chemical structure as well as resulting bonds between the polypropylene and the L-Cys was analyzed using Thermo-Nicolet iS10 spectrophotometer (64 scans; spatial frequency resolution of $4 \mathrm{~cm}^{-1}$ ).

Differential scanning calorimetry (DSC) The differences in crystallinity between the pure polypropylene and the polypropylene grafted with L-Cys were observed by differential scanning calorimetry (DSC 204 Phoenix (Netzsch)). Each sample was placed in aluminum receptacles, followed by an analysis with nitrogen atmosphere ranging from 30 to $300^{\circ} \mathrm{C}$, with a temperature gradient of $2{ }^{\circ} \mathrm{C}$ per minute. The routine procedure was performed in order to eliminate the thermal history of the analyzed samples. Melting point $\left(T_{\mathrm{m}}\right)$, enthalpy of fusion $\left(\Delta H_{\mathrm{m}}\right)$ and onset temperature $\left(T_{\text {onset }}\right)$ results were obtained. The degree of crystallinity of the pure polypropylene substrate, and the blend was calculated according to Eqs. 1 and 2, respectively:

$\chi_{\mathrm{PP}}=\frac{\Delta H_{\mathrm{m}}}{\Delta H_{\mathrm{m}}^{0}} \times 100 \%$

$\chi_{\mathrm{PP}-\mathrm{g}-\mathrm{Cys}}=\frac{\Delta H_{\mathrm{m}}}{\text { Blend fraction } x \Delta H_{\mathrm{m}}^{0}} \times 100 \%$

in which $\chi$ stays for the crystallinity, $\Delta H_{\mathrm{m}}$ is the enthalpy of melting, $\Delta H_{\mathrm{m}}^{0}$ is the reference enthalpy of melting of $100 \%$ crystalline polypropylene.

Scanning electron microscopy (SEM) The polypropylene nanofibers, as well as the nanofibers resulting from the grafting of polypropylene with L-Cys, were observed under SEM (Hitachi S2700). 


\section{Substrate functionalization with Cys-LC-} LL-37 peptide

\section{Minimal inhibitory concentration (MIC) of Cys-LC-LL-37} activity against $P$. aeruginosa

The minimal inhibitory concentration of Cys-LC-LL37 against $P$. aeruginosa was determined using the broth microdilution method, as described on M07-A6 guidelines JIS L 1902:2002, in order to define the best concentration to be used.

Cys-LC-LL-37 was dissolved in sterile $\mathrm{ddH}_{2} \mathrm{O}$ to a final concentration of $10 \mu \mathrm{g} / \mathrm{mL}$ and serially diluted with sterile Mueller-Hinton broth. For each Cys-LCLL-37 dilution, P. aeruginosa was inoculated as liquid culture with its turbidity adjusted to $0.5 \mathrm{McF}$ arland (approximately $1 \times 10^{8} \mathrm{CFU} / \mathrm{mL}$ ). After incubation for $24 \mathrm{~h}$ at $37^{\circ} \mathrm{C}$, antibacterial activity was observed as sediment of dead P. aeruginosa on the bottom of the wells. Control tubes of the medium and broth with Cys-LC-LL-37 were also incubated.

\section{Cys-LC-LL-37 cross-linking onto PP-g-SH surface}

The produced nanofibrous samples underwent dialysis (Medicell Membranes Ltd) in $\mathrm{ddH}_{2} \mathrm{O}$ for 3 days at room temperature while gently stirred to remove any solvent and impurities. The PP-g-SH nanofibers presented thiol groups available on the surface, which were used to covalently bind Cys-LC-LL-37 antimicrobial peptide (AMP) through its thiol group, forming a disulfide bond. For this purpose, all samples and controls were functionalized by an exhaustion process with $2.5 \mu \mathrm{g} / \mathrm{mL}$ of Cys-LC-LL-37 at $45^{\circ} \mathrm{C}$ for $60 \mathrm{~min}$ at $15 \mathrm{rpm}$. Finally, they were washed to remove any unbound/misbound Cys-LCLL-37. In this way, the cationic terminal part of CysLC-LL-37 became exposed in order to eliminate bacteria.

Yield of the cross-linking In order to check how efficient the cross-linking was, Bradford's reagent was used to determine the concentration of Cys-LC-LL-37 in solution before and after the cross-linking. The procedure is based on the formation of a complex between the dye, Coomassie Brilliant Blue $G$ and the amines of Cys-LC-LL-37 in solution. Briefly, $1 \mathrm{~mL}$ of each sample was added to a test tube containing $1 \mathrm{~mL}$ of Bradford's reagent, vortexed, incubated at room temperature for $5 \mathrm{~min}$ and measured spectrophotometrically at $595 \mathrm{~nm}$.

Washing cycles

In order to evaluate the stability of the bounding of Cys-LC-LL-37 and if it resisted to laundry, PP-S-SLC-LL-37 samples were subjected to 25 washing cycles each in fresh $\mathrm{ddH}_{2} \mathrm{O}$, according to procedure adapted from international standard EN ISO 105-C06:2010.

\section{Elemental analysis}

The atomic composition of the functionalized substrate was analyzed by energy-dispersive $\mathrm{X}$-ray spectroscopy (EDS), a device interconnected with SEM.

\section{Antimicrobial activity assessment}

Samples were tested through E 2180-01 Standard Test Method for Determining the Activity of Incorporated Antimicrobial Agents that is designed for testing hydrophobic materials suited for the healthcare industry. Briefly, Staphylococcus aureus and Pseudomonas aeruginosa inocula were prepared from an overnight liquid culture. Bacterial concentrations were then adjusted to $1-2 \times 10^{8}$ cells $/ \mathrm{mL}$ (equivalent to $0.5 \mathrm{McF}$ arland) and working standards prepared to a final concentration of $1 \pm 0.3 \times 10^{5} \mathrm{CFU} / \mathrm{mL}$. Textiles were placed in Falcon tubes to release the bacterial cells from the textile samples after the 24-h incubation period. The resulting suspensions were then plated to determine the viable counts. Antimicrobial activity was calculated in order to determine bacterial growth inhibition (\% of Inhibition) at $24 \mathrm{~h}$ :

\% Inhibition $=\frac{C-A}{C} \times 100$

where $C$ is the average value of colony-forming units (CFU) of controls, and $A$ represents the average value of CFU of PP-S-S-LC-LL-37.

To determine whether the developed substrate has bacteriostatic or bactericidal activity, the following equations were solved:

Bacteriostatic activity $=M_{\mathrm{b}}-M_{\mathrm{c}}$

Bactericidal activity $=M_{\mathrm{a}}-M_{\mathrm{c}}$

where $M_{\mathrm{a}}$ is the $\log 10$ of the average number of bacterial colonies on the control samples at $\mathrm{T} 0, M_{\mathrm{b}}$ is 
the $\log 10$ of the average of bacterial colonies on the control samples at T24 h, and $M_{\mathrm{c}}$ is the $\log 10$ of the average of bacterial colonies on the functionalized samples at T24 h.

\section{Statistical analysis}

Samples were analyzed statistically, and comparisons that had a $p$ value $<0.5$ were considered statistically different. One-way ANOVA and Tukey post Hoc tests for multiple comparisons between all samples were performed with SPSS 21.0.

\section{Results}

\section{Surface-active bulk additive grafting}

\section{Fourier transform infrared spectroscopy (FT-IR)}

Figure 1 depicts the IR peaks relative to the polypropylene. The strong peaks of polypropylene at 2980 and $2971 \mathrm{~cm}^{-1}$ are associated with C-H stretching vibrations of methyl group; $2888 \mathrm{~cm}^{-1}$ is associated with $\mathrm{C}-\mathrm{H}$ stretching vibrations of methylene group. The vibrations at 1463 and $1382 \mathrm{~cm}^{-1}$ are due to methylene and methyl bending, respectively. In addition, medium intensity peaks are observed at $1150 \mathrm{~cm}^{-1}$, which is related to both methyl symmetric and antisymmetric deformation, whereas the one at $952 \mathrm{~cm}^{-1}$ is related to methyl group rocking vibration, respectively.
PP-g-SH after 25 washing cycles was also assessed by FT-IR. IR spectra confirmed L-Cys grafted on PP, as shown by the evidence of the weak intensity peak associated with -SH exposure on PP surface at $2550 \mathrm{~cm}^{-1}$. The peaks observed in the high-frequency region, namely 2950 and $2910 \mathrm{~cm}^{-1}$, are related to the vibration of the alkyl thiol chains. Furthermore, S-H deformation vibration was detected with the peaks 895,839 and $805 \mathrm{~cm}^{-1}$. Also the peaks at 2950 and $2879 \mathrm{~cm}^{-1}$ are related to both methyl asymmetric and symmetric vibrations. These bands (narrow peaks) might be attributed to the alkyl chains of PP crosslinked with the L-Cys.

The peak at $3630 \mathrm{~cm}^{-1}$ is assigned to structural hydroxyl groups of L-Cys, which occupy the empty positions of PP-g-SH.

The peaks at 2888 and $1463 \mathrm{~cm}^{-1}$ assigned to methylene vibrations increased in intensity as crystallinity develops-PP-g-SH-and were less evident with the less crystalline state of PP.

\section{Differential scanning calorimetry (DSC)}

The thermal properties of PP and PP-g-SH were investigated (Fig. 2). The melting temperatures $\left(T_{\mathrm{m}}\right)$ for control and PP-g-SH were obtained from the heating scan. PP showed a $T_{\mathrm{m}}$ of $164.8^{\circ} \mathrm{C}$, while PP-g-SH presented one of $167.42{ }^{\circ} \mathrm{C}$. Furthermore, the crystallinity of PP-g-SH also increased-65.07\%-when compared to PP - 51.39\%. This higher number of connections causes an interconnected pore network and a high surface area per unit mass, which is an advantage to microbial killing. The enthalpy of fusion
Figure 1 IR spectra of native $\mathrm{PP}$ and PP-g-SH.

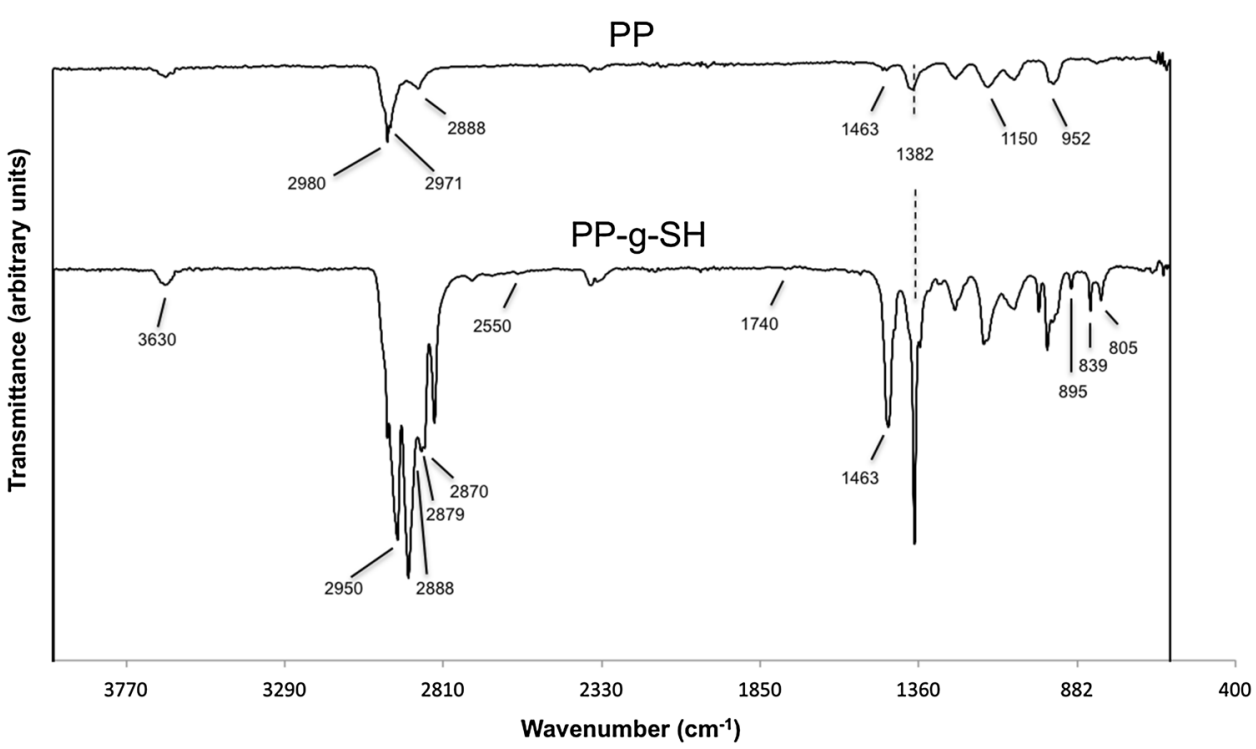


Figure 2 Differential scanning calorimetry spectra of PP and PP-g-SH.

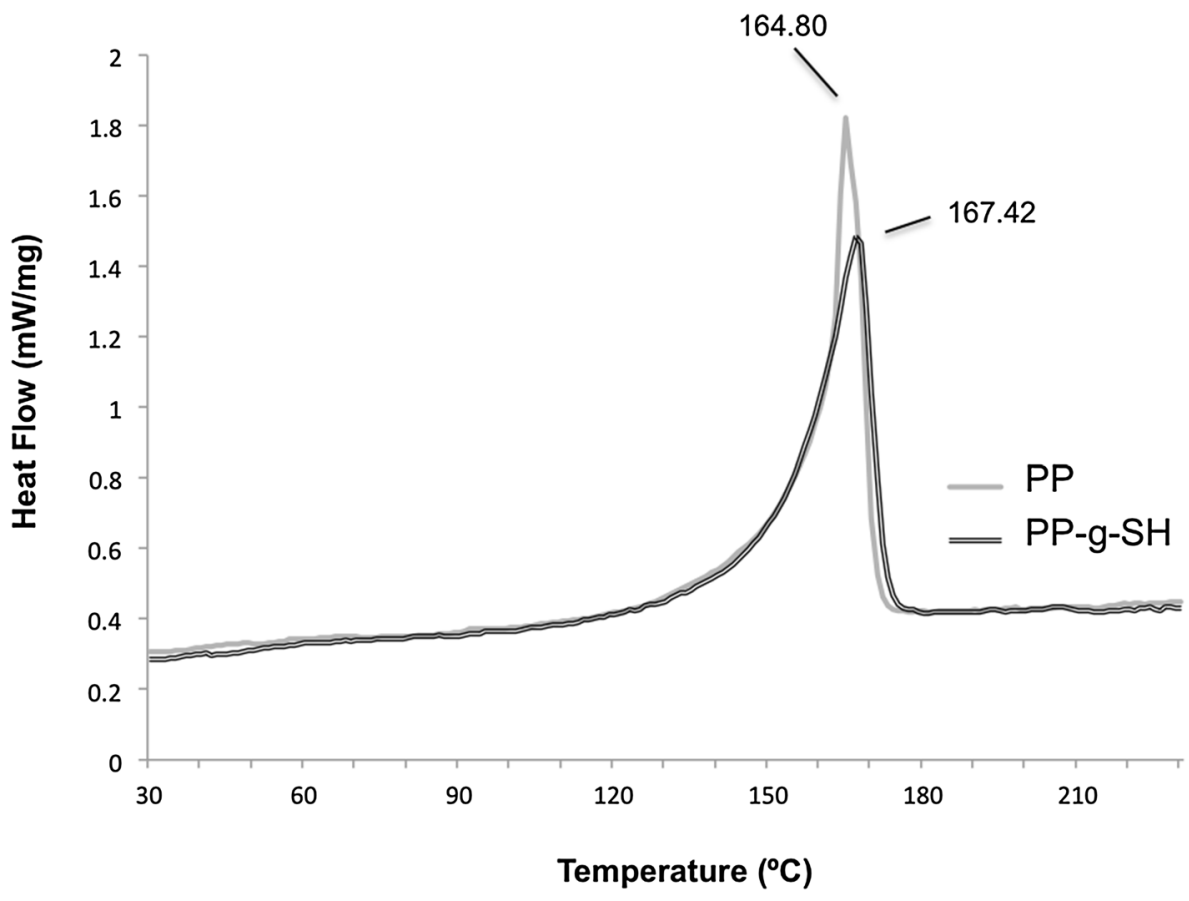

(108.8 J/g) of PP-g-SH was higher than the enthalpy of fusion for PP $(107.4 \mathrm{~J} / \mathrm{g})$.

\section{Scanning electron microscopy (SEM)}

The topography of PP and PP-g-SH nanofibers was observed by SEM microscopy, and their structural differences are shown in Fig. 3. The PP-g-SH condition revealed higher number of nanofibers per area with increased cross-linking between them, when comparing to PP nanofibers. Hence, PP-g-SH exhibited an increased surface area per mass.

\section{Substrate functionalization}

\section{Yield of the cross-linking}

The concentration of Cys-LC-LL-37 in solution before and after its cross-linking on PP-g-SH resulted in the cross-linking efficiency of $95.30 \%$.

\section{Minimal Inhibitory concentration (MIC) of Cys-LC-LL-37 activity against $P$. aeruginosa}

The minimal inhibitory concentration (MIC) value for $P$. aeruginosa was $5 \mu \mathrm{g} / \mathrm{mL}$.

\section{Energy-dispersive X-ray spectroscopy (EDS)}

EDS analysis allowed us to analyze the atomic elements of the PP, PP-g-SH and PP-S-S-LC-LL-37 samples. The fact that polypropylene does not present sulfur in its native structure allowed us to easily compare the results of the modifications that were made. The first modification of the polypropylene, made by surface modifying additives grafting, generated thiol groups on the surface of the nanofibers (PP-g-SH), at $0.10 \%$ wt.(percentage by weight). The coating of Cys-LC-LL-37 on thiol groups yielded PPS-S-LC-LL-37. PP-S-S-LC-LL-37 amount of sulfur was $0.21 \mathrm{wt} \%$. In conclusion, for each sulfur detected in PP-g-SH, there was one Cys-LC-LL-37 bound, which means a very high cross-linking efficiency.

\section{Antimicrobial activity assessment}

The E 2180-01 Standard Test Method for Determining the Activity of Incorporated Antimicrobial Agent(s) in Polymeric or Hydrophobic Materials was followed to determine the viability reduction with the application of the PP, PP exhausted with Cys-LC-LL37, PP-g-SH and PP-S-S-LC-LL-37. The antibacterial efficiency of these samples was quantitatively evaluated against $S$. aureus and P. aeruginosa (Fig. 4).

Following 25 washing cycles, the assay showed for S. aureus that after $24 \mathrm{~h}$ of contact with PP-S-S-LC-LL- 

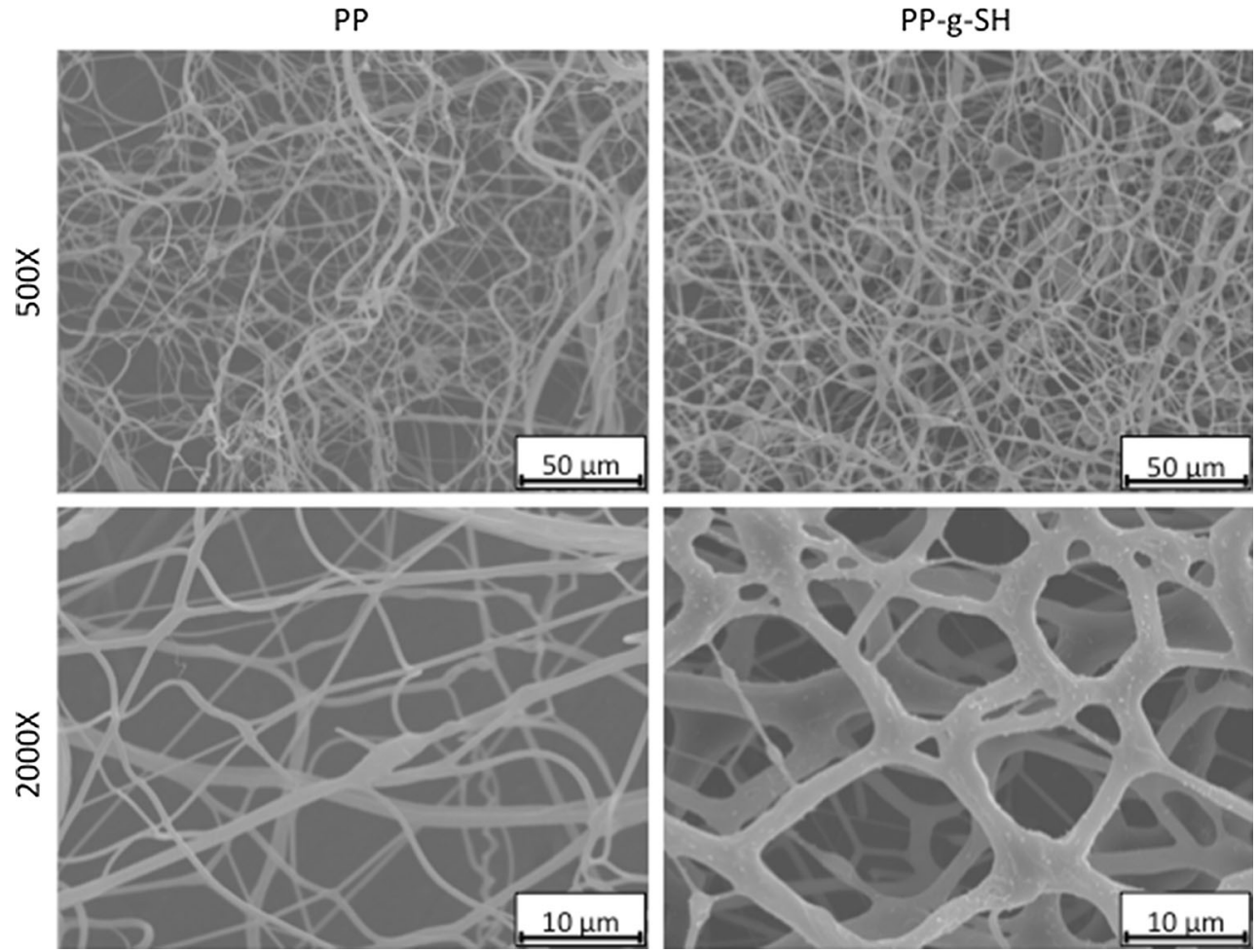

Figure 3 SEM images of 15\% (w/v) PP and PP-g-SH. These nanofibers were electrospun from NanoSpider.
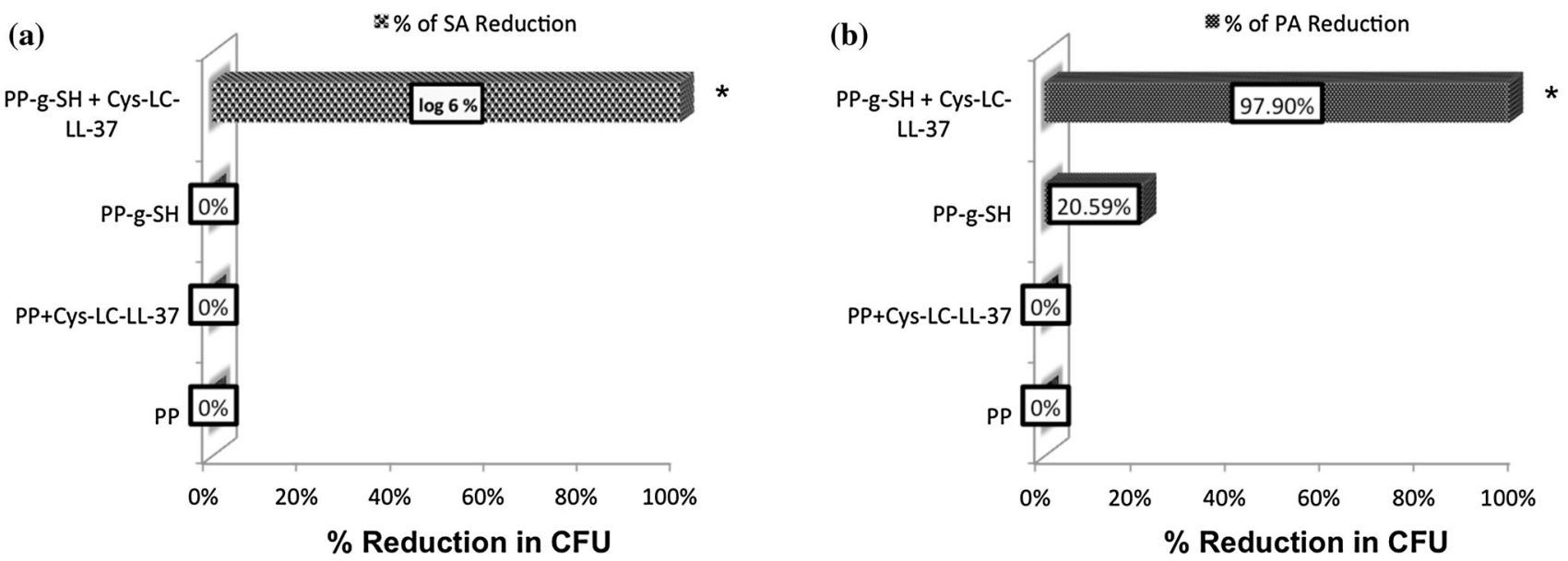

Figure 4 Percentage of microbial inhibition against S. aureus (a) and P. aeruginosa (b). Values were derived from results of native PP. * Statistically significant $(p<0.05)$.

37, there was a total reduction in their viability of 99.9999\% in comparison with the PP, as shown in Fig. 4a. For P. aeruginosa after $24 \mathrm{~h}$ of contact with the same condition-PP-S-S-LC-LL-37-there was a remarkable reduction in viability of $97.41 \%$ in comparison with the PP (Fig. 4b). However, when thiol groups were exposed at the surface of PP, they were unable to inhibit the growth of $S$. aureus at all, but $20.59 \%$ of $P$. aeruginosa. As expected, the condition in which PP was exhausted with Cys-LC-LL-37 had no reactive groups to form any linkage, hence no microbial reduction was observed for both strains. It is possible to observe that the percentages of viability reduction were the highest and significant for PP-S-SLC-LL-37, which translates the stability of the disulfide bond between PP-g-SH and Cys-LC-LL-37 throughout 25 washing cycles and the effectiveness of the antimicrobial peptide Cys-LC-LL-37. 
According to Eqs. 4 and 5, for S. aureus - - $0.09-$ and $P$. aeruginosa $-0.16-$, there was no bacteriostatic effect of PP-g-SH, but a bactericidal effect of PP-S-SLC-LL-37, for both S. aureus - 3.52 - and P. aeruginosa - 1.68. This meets JIS L 1902, which states that the bacteriostatic activity of a sample cannot be inferior to 2 and the bactericidal value cannot be inferior to 0 .

\section{Discussion}

Due to the high number of patients afflicted, our approach was rather on an application that would act toward the prevention of microorganism establishment in garment and, in this way, a consequent eradication of these microorganisms in the hospital air. It is important to underline that polypropylene fibers are the most used in hospital garments either as surgical protective clothing as patient disposable garments used in clinical settings. Therefore, this investigation is of the utmost importance since it is the first to report novel ways of having polypropylene garments with an added antimicrobial function. Our investigations led to the development of a garment with thiol hooks, which allowed the functionalization with the antimicrobial peptide Cys-LC-LL37 through its cysteine thiol. In this way, we sought to obtain an application resistant to multiple washing cycles, namely whether the disulfide bond formed would be resistant to 25 washing cycles and if CysLC-LL-37 would be intact and equally bioactive after these washing cycles had been performed.

At first we looked at how far we could modify polypropylene through modification by L-Cys and produce nanofibers by electrospinning, other than by the extrusion technique that would most probably deactivate the activity of the biomolecules. The production of nanofibers was successful, both for PP (polypropylene) and PP-g-SH (PP grafted with LCys), yielding the latter ones more conspicuous nanofibers and in a greater number, as compared to polypropylene control. The results of FT-IR and EDS showed that PP-g-SH was exhibiting thiol groups at the surface of its nanofibers. In addition, DSC showed an increase in $T_{\text {fusion, }}$ which meant that stronger bonds were formed as well as higher number of cross-links. As consequence of stronger bonds, an increase in crystallinity was also observed. Furthermore, this higher number of cross-linking caused an interconnected pore network and a larger surface area per unit mass, which is an improvement to microbial killing. Moreover, when PP melted, dissolved and combined with L-Cys, its decreased enthalpy of fusion $(1.48 \mathrm{~mW} / \mathrm{mg})$ might be due to weak intermolecular forces and the grafting reactions between PP and L-Cys, where PP chain was split into parts to combine with L-Cys to produce PP-g-SH. The enthalpy of fusion for PP was $1.82 \mathrm{~mW} / \mathrm{mg}$.

These data led us to test, in a second approach, whether (i) the thiol hooks exposed on the modified polypropylene (PP-g-SH) were capable of exerting an antimicrobial effect themselves or, on the other hand, (ii) the coating of the antimicrobial peptide Cys-LCLL-37 between thiols would be a better strategy for this application, in terms of antimicrobial effect. The coating of Cys-LC-LL-37 over PP-g-SH had a very high cross-linking efficiency, which accounted for $95.30 \%$. The results provided by elemental analysis (EDS) of the aforementioned surfaces indicated the presence of the double of sulfur atoms in the samples PP-S-S-LC-LL-37, when compared to PP-g-SH. This confirms the success of the coating between PP-g-SH and Cys-LC-LL-37, since all thiol groups of PP-g-SH were mainly occupied bonding the Cys of the antimicrobial peptide. While PP-g-SH showed to exert no bacteriostatic effect ( $0 \%$ for S. aureus; $20.59 \%$ for $P$. aeruginosa), the Cys-LC-LL-37 coating on PP-g$\mathrm{SH}$, with half of the concentration of MIC obtained for both bacteria, induced a bactericidal response (99.9999\% for SA; $97.90 \%$ for PA) after 25 washing cycles. We have also discovered, at the test-control level, whether mixing of PP with Cys-LC-LL-37 would lead to the formation of any bond and, as expected, it was found that Cys-LC-LL-37 did not cross-link to any nanofiber of polypropylene, either by EDS that showed no traces of sulfur $-0 \mathrm{wt} \%$ - or by antimicrobial assays that showed no microbial reduction. This explains why the presence of the thiol hooks is odd to establish the resistant disulfide bond when linking to Cys-LC-LL-37. We also found from EDS results and antimicrobial assays that Cys-LC-LL37 was not released from the PP-g-SH surface, regardless of the washing cycles performed, up to 25. Furthermore, due to the fact that Cys-LC-LL-37 is charged and presents hydrophilic portions separated from the hydrophobic areas, proved advantageous over only thiolated groups. A thiolated surface with no spacer that could distance $-\mathrm{SH}$ from surface of nanofibers did not confer flexibility and exerted no bacteriostatic or bactericidal effect. 
The release of toxic substances from a textile product with skin contact is a precondition for the formation of skin irritations. However, examination on cytotoxicity of components used separately in previous works $[22,23,28]$ permitted the evaluation of the danger potential for a skin irritation. Cys-LC-LL-37 was tested against normal human dermal fibroblasts, the results of which showed no growth variation greater than $30 \%$, which means that this antimicrobial peptide is not cytotoxic and can be used for skin contact [28]. The testing of cytotoxicity of L-Cys on connective tissue cells L 929 also showed a growth variation inferior to 30\% [29]. Polypropylene showed no cytotoxicity when its extracts were blended in plasma [30], where it was shown that there was no difference on viability and cell adhesiveness of chickembryo fibroblasts. Also, the morphology of calfkidney fibroblasts was also normal [30]. The three agents used in this study are therefore considered to be safe agents.

Thus, considering previous research and the potential of electrospinning for the development of nanofibers for use in antimicrobial garments, we intended to develop a new garment to kill microorganisms and thereby control the safety of hospital environment. It is thus expected that a garment free of microorganisms will not promote their spread into the hospital environment.

\section{Conclusions}

This study addresses the potential of L-cysteine amino acid to graft polypropylene chain, so as to thread nanofibers through electrospinning. These nanofibers provided a novel, smoother and increased surface-to-area substrate with thiol group hooks, which could be used to bind the antimicrobial peptide Cys-LC-LL-37 through disulfide bonding.

The skin of patients with diabetes or immunodeficiency is particularly susceptible to infection by different microorganisms. The opportunistic pathogens $S$. aureus and $P$. aeruginosa are the agents responsible for some lethal infections under hospital settings. In this way, our application sought to impede the dispersal of pathogens to the air, which should minimize their settling and reduce the chances of cross infection.

The grafting of L-Cys on polypropylene did not provide any significant antibacterial activity. Instead, its free thiol groups promoted an effective means of cross-linking to Cys-LC-LL-37, which dramatically reduced S. aureus by $99.9999 \%(\log 6)$ and P. aeruginosa by $97.90 \%$.

In this regard, a new material, safe, with strong antimicrobial properties and alternative to antibiotics is herein presented for potential use as garment under hospital settings.

\section{Funding}

The authors acknowledge the Fundação para a Ciência e Tecnologia (FCT) for the PhD Grant SFRH/ BD/91444/2012 and Programa Operacional Capital Humano (POCH) and European Union for co-funding the work.

\section{Compliance with ethical standards}

Conflict of interest The authors declare no conflicts of interest.

\section{References}

[1] Pinon A, Gachet J, Alexandre V, Decherf S, Vialette M (2013) Microbiological contamination of bed linen and staff uniforms in a hospital. Adv Microbiol 3:515-519

[2] Chemaly RF, Simmons S, Dale C, Ghantoji SS, Rodriguez M, Gubb J, Stachowiak J, Stibich M (2014) The role of the healthcare environment in the spread of multidrug-resistant organisms: update on current best practices for containment. Therap Adv Infect Dis 2(3-4):79-90

[3] http://www.lenzing.com/en/investors/equity-story/global-fibermarket.html. Accessed 12 July 2017

[4] Statista (2017) https://www.statista.com/statistics/271651/ global-production-of-the-chemical-fiber-industry/. Accessed 12 July 2017

[5] Gashti MP, Willoughby J, Agrawal P (2011) Surface and bulk modification of synthetic textiles to improve dyeability. In: Prof. Peter Hauser (ed) Textile dyeing. InTech, ISBN: 978-953-307-565-5

[6] Fischer-Colbrie G, Heumann S, Liebminger S, Almansa E, Cavaco-Paulo A, Guebitz GM (2004) New enzymes with potential for PET surface modification. Biocatal Biotransform 22(5/6):341-346

[7] Silva CM, Carneiro F, O'Neill A, Fonseca LP, Cabral JSM, Guebitz G, Cavaco-Paulo A (2004) Cutinase- a new tool for biomodification of synthetic fibers. J Polym Sci Part A Polym Chem 22(11):2448-2450 
[8] PETROCHEMICALS \& EPCA - A PASSIONATE JOURNEY. EPCA The European Petrochemical Association A.I.S.B.L./I.V.Z.W. 2016

[9] Karpian HG (2003) Handbook of polypropylene and polypropylene composites. Plastics Engineering. CRC Press, Boca Raton

[10] The Review on Antimicrobial Resistance, chaired by Jim O'Neill. Antimicrobial Resistance: Tackling a crisis for the health and wealth of nations. Dec 2014

[11] Appelbaum PC (2007) Microbiology of antibiotic resistance in Staphylococcus aureus. Clin Infect Dis 45(Supplement 3):S165-S170

[12] Livermore DM (2002) Multiple mechanisms of antimicrobial resistance in Pseudomonas aeruginosa: our worst nightmare? Clin Infect Dis 34(5):634-640

[13] Sun G (2016) Antimicrobial textiles, 1st edn. Woodhead Publishing, Cambridge

[14] Babu KM, Ravindra KB (2015) Bioactive antimicrobial agents for finishing of textiles for health care products. J Text Inst 106(7):706-717

[15] Belyaev EY (2000) Drug synthesis methods and manufacturing technology, new medical materials based on modified polysaccharides (Review). Pharm Chem J 34(11):607-612

[16] Haufe H, Muschter K, Siegert J, Bottcher H (2008) Bioactive textiles by sol-gel immobilised natural active agents. J SolGel Sci Technol 45:97-101

[17] Hori Y, Sato S, Hatai A (2006) Antibacterial activity of plant extracts from azuki beans (Vigna angularis) in vitro. Phytother Res 20:162-164

[18] Fox JL (2013) Antimicrobial peptides stage a comeback. Nat Biotechnol 31:379-382

[19] Nogueira F, Vaz J, Mouro C, Piskin E, Gouveia IC (2014) Covalent modification of cellulosic-based textiles: a new strategy to obtain antimicrobial properties. Biotechnol Bioprocess Eng 19(3):526-533
[20] Pedrosa M, Mouro C, Nogueira F, Vaz J, Gouveia I (2014) Comparison of the antibacterial activity of modified-cotton with Magainin I and LL-37 with potential as wound-dressings. J Appl Polym Sci 131:40997

[21] Nogueira F, Gomes A, Gouveia IC (2016) New garment proposal for prevention of spreading Gram-negative bacteria resistant to carbapenem antibiotic class under hospital settings. J Ind Text. https://doi.org/10.1177/1528083716682919

[22] Nogueira F, Granadeiro L, Mouro C, Gouveia IC (2015) Antimicrobial and antioxidant surface modification toward a newsilk-fibroin (SF)-L-cysteine material for skin disease management. Appl Surf Sci 364:552-559

[23] Nogueira F, Gouveia IC (2017) Amino acid based material for the complementary therapy of Decubitus Ulcers. J Microbiol Biotechnol 27(4):747-758

[24] Grubor B, Meyerholz DK, Ackermann MR (2006) Collectins and cationic antimicrobial peptides of the respiratory epithelia. Vet Pathol 43(5):595-612

[25] Mygind PH, Fischer RL, Schnorr KM et al (2005) Plectasin is a peptide antibiotic with therapeutic potential from a saprophytic fungus. Nature 437:975-980

[26] Weast HRC, Selby SM (1962) Handbook of chemistry and physics, 42nd edn. CRC Press, Cleveland

[27] Anfinsen CB, Bailey K, Anson ML, Edsall JT (1963) Adv Protein Chem 17:iii-xiv, 1-412

[28] Mouro C, Pedrosa M, Vaz J, Gouveia I (2014) Two surface activation strategies to functionalize cotton fibers with CysLC-LL-37 antibacterial peptide. AATCC J Res 1(6):27-33

[29] Caldeira E, Piskin E, Granadeiro L, Silva F, Gouveia IC (2013) Biofunctionalization of cellulosic fibres with L-cysteine: assessment of antibacterial properties and mechanism of action against Staphylococcus aureus and Klebsiella pneumoniae. J Biotechnol 168:426-435

[30] Conning DM, Firth J (1969) Toxicity of polypropylene in tissue culture. Food Cosmet Toxicol 7:461-472 